\title{
CARDINAL FUNCTIONS ON MODIFICATIONS OF UNIFORM SPACES AND FINE UNIFORM SPACES
}

\author{
VĚRA KƯRKOVÁ
}

\begin{abstract}
The paper studies the question for which modifications $r$ of Unif the following theorem can be generalized by substituting a precompact modification $p$ by $r$ : A uniform space has the finest uniformity inducing its proximity if and only if each proximally continuous mapping from this space to any other uniform space is uniformly continuous. By means of two cardinal functions defined on the class of all modifications of Unif there is shown that this is possible only for cardinal modifications $p^{\alpha}$. Assuming GCH, the problem for cardinal modifications $p^{\alpha}$ is solved for uniform spaces of a limited point-character (in dependence on $\alpha$ ).
\end{abstract}

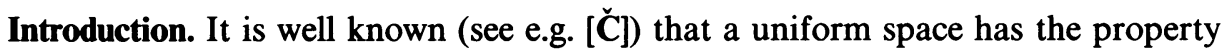
that each proximally continuous mapping from this space to any other uniform space is uniformly continuous if and only if it has the finest uniformity inducing its proximity (precompact modification). Both notions appearing in this equivalence had been generalized by $Z$. Frolik in $\left[F_{1}, F_{2}\right]$. He had defined for a modification (= reflection preserving underlying sets) $r$ of any concrete category the notions of $r$-fine and $r$-simply fine object. Let us recall here these notions for the category Unif of uniform spaces. A uniform space is called $r$-fine if for any uniformly continuous mapping $f: X \rightarrow r Y$ with $Y$ arbitrary, $f: X \rightarrow Y$ is uniformly continuous, too. A uniform space is called $r$-simply fine if it has the finest uniformity inducing its $r$-modification. Evidently each $r$-fine space is $r$-simply fine. The present paper studies the question for which modifications of Unif the opposite implication is also true. A sufficient condition for equality of both classes and some examples of modifications of Unif for which these classes differ are found in the author's paper $\left[\mathbf{K}_{\mathbf{1}}\right]$. A combinatorial problem from $\left[\mathbf{K}_{\mathbf{1}}\right]$ connected with the problem of whether (assuming $\mathrm{GCH}$ ) for each nonlimit infinite cardinal $\alpha$ the classes of $p^{\alpha}$-fine and $p^{\alpha}$-simply fine spaces differ in the category of all zero-dimensional uniform spaces has been solved by V. Rödl in [ $\mathbf{R}_{\mathbf{1}}$ ]. Some conditions on a modification $r$ of Unif which imply the existence of an $r$-simply fine uniform space which is not $r$-fine were formulated in [ $\left.\mathbf{K}_{\mathbf{2}}\right]$.

The present paper has two parts. In the first part, conventions and related results are introduced that will be of later use. Also two cardinal functions on modifications of Unif are introduced and some of their properties are stated. In the second part, it is shown that for any noncardinal modification of Unif, a generalization of the

Received by the editors June 18, 1980 and, in revised form, April 30, 1981.

1980 Mathematics Subject Classification. Primary 54E15; Secondary 54E05.

Key words and phrases. Uniform space, modification, cardinal modification, point-character. 
above theorem is not possible. Further (assuming GCH), for each nonlimit cardinal $\alpha$ a non- $p^{\alpha}$-fine uniform space is constructed which is $p^{\alpha}$-simply fine with respect to uniform spaces of a point-character less than or equal to $c f \beta$, where $\beta^{+}=\alpha$.

I. For the most part we will use the terminology and notation of [I]. In particular a uniform space is what is sometimes called a separated uniform space. By $|X|$ we denote the underlying set of a uniform space $X$. For two uniform spaces $X$ and $Y$ with the same underlying sets we write $X \leqslant Y$ if the identical mapping from $X$ to $Y$ is uniformly continuous. If $|X|=|Y|$ and $X \leqslant Y$ we say that $X$ is finer than $Y$ and $Y$ is coarser than $X$. A uniform space is called zero-dimensional if it has a basis formed by partitions. For any infinite cardinal $\alpha$ we denote by $D(\alpha)$ a uniformly discrete uniform space with an underlying set of cardinality $\alpha$. The point-character of a uniform space $X$ is the minimal infinite cardinal $\alpha$ such that $X$ has a basis of uniform coverings with the property that each point $x \in X$ is a member of less than $\alpha$ members of this covering. Recall that the problem of [I], whether there exists a uniform space with the point-character greater than $\boldsymbol{\aleph}_{0}$, has been solved by Šcepin and Pelant. Pelant proved e.g. that for every cardinal $\alpha \geqslant \boldsymbol{N}_{1}$ the point-character of $l_{\infty}(\alpha)$ is greater than $\boldsymbol{\aleph}_{0}$ (see [P]). A great deal of work on spaces with a large point-character has been made by Pelant and Rödl (see [P-R, $\left.\mathbf{R}_{2}, \mathbf{R}_{3}\right]$ ).

For the terminology concerning category theory see e.g. [M]. By Unif we denote the category of all uniform spaces and uniformly continuous mappings. Recall that a full subcategory of Unif is reflective if and only if it is closed under the formation of products and subspaces (dually coreflective if and only if it is closed under the formation of sums and quotients). A reflection (coreflection resp.) is called modification (comodification) if it preserves underlying sets. Recall that each coreflection of Unif is a comodification (see [K]) and contains all uniformly discrete uniform spaces.

The cardinal modification $p^{\alpha}$, where $\alpha$ is an arbitrary infinite cardinal, assigns (assuming $\mathrm{GCH}$ ) to any uniform space the space $p^{\alpha} X$ generated by all the uniform coverings of $X$ of a cardinality less than $\alpha$. The assumption of $\mathrm{GCH}$ in the definition of $p^{\alpha}$ is necessary for every $\alpha \geqslant \boldsymbol{\aleph}_{2}$ as it was shown by J. Pelant. For $\alpha=\boldsymbol{\aleph}_{0}$ or $\alpha=\aleph_{1}$ the system of all the uniform coverings of the cardinality less than $\alpha$ of any uniform space $X$ forms a uniformity. But for every $\alpha \geqslant \aleph_{2}$, the assumption that the system of all uniform coverings of cardinality less than $\alpha$ of any uniform space $X$ forms a uniformity is consistent with and independent on ZFC (see [P]). For the usual precompact modification we use the usual notation $p$ instead of $p^{\aleph_{0}}$.

If $r_{1}, r_{2}$ are any two modifications of Unif, we write $r_{1} \leqslant r_{2}$ if $r_{1} X \leqslant r_{2} X$ for every uniform space $X$. Recall from $\left[\mathbf{V}_{1}\right]$ that for any modification $r$ of Unif we have $r \leqslant p$.

For any modification $r$ of Unif we denote by $r^{f}$ the class of all $r$-fine uniform spaces ( $r$-fine uniform spaces are defined in the introduction). It is easy to verify that each class $r^{f}$ is closed under the formation of sums and quotients and hence coreflective.

For any nonidentical modification $r$ of Unif, define $\mu_{r}=\min \left\{\right.$ card $\left.|X| \mid X \notin r^{f}\right\}$. Notice that for any uniform space $X, X \neq r X$ implies $X \notin r^{f}$. Hence for any nonidentical modification $r$ of Unif the class $\left\{X \in\right.$ Unif, $\left.X \notin r^{f}\right\}$ is nonempty. For an identical modification id put $\mu_{\text {id }}=\infty$, and extend the ordering $<$ of the class $\mathrm{Cn}$ 
of all cardinals to $\mathrm{Cn} \cup\{\infty\}$ by $\alpha<\infty$ for every $\alpha \in \mathrm{Cn}$. Notice that if $r_{1}, r_{2}$ are arbitrary modifications of Unif with $r_{1} \leqslant r_{2}$ then $\mu_{r_{1}} \geqslant \mu_{r_{2}}$. Hence for every modification $r$ of Unif the value of $\mu_{r}$ is infinite since $\mu_{r} \geqslant \mu_{p}=\boldsymbol{\kappa}_{0}$.

For an arbitrary modification $r$ of Unif, define $\delta_{r}=\min \left\{\alpha \in \mathrm{Cn} \mid \alpha \geqslant \omega_{0}\right.$ and $r D(\alpha) \neq D(\alpha)\}$ if there exists an infinite cardinal $\alpha$ such that $r D(\alpha) \neq D(\alpha)$ and $\delta_{r}=\infty$ otherwise. As for $\mu$, for $\delta$ we have also $\delta_{r_{1}} \geqslant \delta_{r_{2}}$ whenever $r_{1} \geqslant r_{2}$.

The following proposition shows that the cardinal function $\delta_{r}$ describes the behavior of $r$ on zero-dimensional uniform spaces. It is a generalization of a proposition from $\left[\mathbf{V}_{2}\right]$.

Proposition I.1. For every modification $r$ of Unif and every zero-dimensional uniform space $X$ we have $r X=p^{\delta_{r}} X$ if $\delta_{r}<\infty$ and $r X=X$ if $\delta_{r}=\infty$.

Proof. First suppose that $\delta_{r}=\infty$. Given a uniform partition $\mathcal{U}$ of $X$, choose a uniformly continuous mapping $f: X \rightarrow D$ such that $थ=\left\{f^{-1}(d) \mid d \in D\right\}$ and $D$ is uniformly discrete. Since $r D=D$ (even for $D$ finite, since every finite $D$ is a retract of $D\left(\aleph_{0}\right)$ ), we have $थ \in r X$.

Now suppose that $\delta_{r}<\infty$. Then $r X$ contains no uniform partition of cardinality $\delta_{r}$, since otherwise $D\left(\delta_{r}\right)$ would be a subspace of $r X$. But this would contradict the fact that $r D\left(\delta_{r}\right) \neq D\left(\delta_{r}\right)$. So $r X=p^{\delta_{r} r} X \geqslant p^{\delta_{r}} X$. To prove that $r X=p^{\delta_{r}} X$, choose any partition $\mathfrak{V} \in X$ of cardinality less than $\delta_{r}$ and a uniformly continuous mapping $h: X \rightarrow D$ such that $\mathfrak{V}=\left\{h^{-1}(d) \mid d \in D\right\}$, where $D$ is uniformly discrete with card $|D|<\delta_{r}$. Since $r D=D$, we have $\mathfrak{V} \in r X$.

Recall from $\left[\mathbf{V}_{2}\right]$ that for any modification $r$ of Unif there exists a finest embedding preserving modification $r_{e}$ coarser than $r$. For any uniform space $X$ and any uniform embedding of $X$ into an injective uniform space $J, r_{e} X$ is a subspace of $r J$.

LEMMA I.2. For every modification $r$ of Unif, $r^{f}=\left(r_{e}\right)^{f}$.

Proof. $\left(r_{e}\right)^{f} \subseteq r^{f}$, since $r_{e} \geqslant r$. Let $X \in r^{f}, Y$ be an arbitrary uniform space and $f$ : $X \rightarrow r_{e} Y$ an arbitrary uniformly continuous mapping. Let $j: Y \rightarrow J$ be an embedding into an injective uniform space $J$. Since $r_{e} J=r J, j \circ f: X \rightarrow J$ is uniformly continuous and hence $f: X \rightarrow Y$ is uniformly continuous, too.

Lemma I.2 shows that $\mu_{r}=\mu_{r_{e}}$ for every modification $r$ of Unif.

II. For every modification $r$ of Unif we have $\mu_{r} \leqslant \delta_{r}$, since $r D(\alpha) \neq D(\alpha)$ implies $D(\alpha) \notin r^{f}$. The next theorem shows that the equality of these two cardinal functions is necessary for the validity of the generalization of the theorem quoted in the introduction.

THEOREM II.1. Let $r$ be a modification of Unif with $\delta_{r}>\mu_{r}$. Then there exists an $r$-simply fine uniform space which is not $r$-fine. 
Proof. Take any non-r-fine uniform space $Y$ with card $|Y|=\mu_{r}$. Denote by $P_{Y}$ the uniform space with an underlying set $\{(x, y, 0) \mid x, y \in Y$ and $x \neq y\} \cup$ $\{(x, y, 1) \mid x, y \in Y$ and $x \neq y\}$ and a basis of a uniformity generated by all the covers

$$
\begin{aligned}
\mathscr{P}_{\mathcal{Q}}= & \{\{(x, y, 0),(x, y, 1)\} \mid x \in \operatorname{St}(y, \mathcal{Q}), x, y \in Y \text { and } x \neq y\} \\
& \cup\{\{(x, y, 0)\},\{(x, y, 1)\} \mid x \notin \operatorname{St}(y, \mathcal{Q}), x, y \in Y\},
\end{aligned}
$$

where $\mathscr{Q} \in Y$. It is stated in [I, p. 52] that $Y$ is a uniform quotient of $P_{Y}$. Since $r^{f}$ is coreflective, $P_{Y}$ cannot be $r$-fine. We will now verify that $P_{Y}$ is $r$-simply fine. $P_{Y}$ is zero-dimensional, so by Proposition I.1 $r P_{Y}=p^{\delta_{r}} P_{Y}$. Since card $\left|P_{Y}\right|=\operatorname{card}|Y|=$ $\mu_{r}<\delta_{r}$, it follows that $r P_{Y}=P_{Y}$. Then the coverings

$$
\begin{aligned}
\Re_{\mathscr{V}} & =\{\{(x, y, 0),(x, y, 1)\} \mid(x, y, 0) \in \operatorname{St}((x, y, 1), \mathscr{V}), x, y \in Y \text { and } x \neq y\} \\
& \cup\{\{(x, y, 0)\},\{(x, y, 1)\} \mid(x, y, 0) \notin \operatorname{St}((x, y, 1), \mathscr{V}), x, y \in Y \text { and } x \neq y\}
\end{aligned}
$$

with $\mathscr{V} \in X$ form a basis of $X$ because of the inequality

$$
\mathcal{V} \wedge\{\{(x, y, 0),(x, y, 1)\} \mid x, y \in Y \text { and } x \neq y\}<\mathscr{R}_{\mathcal{V}}<\mathscr{V}
$$

Hence $X$ is zero-dimensional and we may use Proposition I.1 to get $r X=p^{\delta_{r}} X$. Since card $|X|=\operatorname{card}\left|P_{Y}\right|<\delta_{r}$, we have $p^{\delta_{r}} X=X$. So $X=r X=r P_{Y}=P_{Y}$.

We immediately obtain from Theorem II. 1 that the classes of $r$-fine and $r$-simply fine spaces are distinct for any nonidentical modification with $\delta_{r}=\infty$. There are many such $r$, e.g. the four modifications described in [I, p. 69] given by the following combinatorial properties of a covering: point-finiteness, star-finiteness, starboundedness, or finite dimensionality. But Theorem II.1 has stronger consequences. The following two propositions together with Theorem II.1 will show that the classes of $r$-fine and $r$-simply fine spaces are distinct for any noncardinal modification $r$.

Proposition II.2. Let $r$ be an arbitrary modification of Unif with $\delta_{r}<\infty$. Then $r \geqslant p^{\delta_{r}}$.

Proof. By the same reasoning as in the proof of Proposition I.1 (the case of $\left.\delta_{r}<\infty\right)$ we obtain that $r \geqslant p^{\delta_{r}}$.

PROPOSITION II.3. Let $r$ be a nonidentical embedding preserving modification of Unif. Then $r \leqslant p^{\mu}$.

Proof. We will show that if $X$ is a uniform space with $X=p^{\mu_{r}} X$, then $X=r X$. It is well known that each uniform space $X$ with $X=p^{\mu_{r}} X$ is projectively generated by a family of metric spaces having dense subsets of cardinalities less than $\mu_{r}$. It will suffice to show that if $M$ has a dense subspace $S$ of a cardinality less than $\mu_{r}$, then it follows from the definition of $\mu_{r}$ that $r S=S$. Since $r$ is embedding preserving, the continuous identity mapping $1: r M \rightarrow M$ is uniformly continuous on a dense subspace $r S$. Hence 1: $r M \rightarrow M$ is uniformly continuous.

COROllaRY II.4. Let $r$ be a nonidentical modification of Unif with $\mu_{r}=\delta_{r}$. Then $r=p^{\mu_{r}}=p^{\delta_{r}}$. 
Proof. Since $\mu_{r}=\mu_{r_{e}}$ by Lemma I.2, we have by Propositions II.2 and II.3 $p^{\delta_{r}} \leqslant r \leqslant r_{e} \leqslant p^{\mu_{r}}$ for an arbitrary nonidentical modification $r$ with $\delta_{r}<\infty$. So if $\mu_{r}=\delta_{r}$, we have $p^{\delta_{r}}=r=r_{e}=p^{\mu_{r}}$.

The preceding results show that the characterization of the modifications $r$ of Unif for which the classes of $r$-fine and $r$-simply fine spaces coincide reduces to the consideration of cardinal modifications. It was stated in the introduction that for a precompact modification both classes are equal. This result has been generalized (assuming $\mathrm{GCH}$ ) in the author's paper $\left[\mathbf{K}_{1}\right]$ to any cardinal modification $p^{\alpha}$ with $\alpha$ a limit cardinal. (Recall the idea of the proof: First one must prove the proposition stating that for every embedding preserving modification $r$ of Unif with the property that $r(X \wedge r Y)=r X \wedge r Y$ for every two uniform spaces $X$ and $Y$ with the same underlying sets $(\wedge$ denotes infimum w.r.t. the above defined ordering $\leqslant)$ the classes of $r$-fine and $r$-simply fine spaces coincide. Then it suffices to verify (assuming $\mathrm{GCH}$ ) that every cardinal modification $p^{\alpha}$ with $\alpha$ limit fulfills the assumptions of this proposition.) For nonlimit cardinals the situation is much more complicated. As we will show in the remainder of the paper (assuming GCH), for each nonlimit cardinal $\alpha=\beta^{+}$there exists a non- $p^{\alpha}$-fine uniform space which is $p^{\alpha}$-simply fine with respect to uniform spaces having point-character less than or equal to $c f \beta$.

THEOREM II.5 (GCH). Let $\beta$ be an infinite cardinal and $\alpha=\beta^{+}$. Then there exists a non-p ${ }^{\alpha}$-fine uniform space $S_{\alpha}$ such that $S_{\alpha}$ is the finest uniform space with point-character less than or equal to cf $\beta$ which has the $p^{\alpha}$-modification equal to $p^{\alpha} S_{\alpha}$.

Proof. Denote by $S_{\alpha}$ a uniform space with the underlying set $\beta \times \alpha$ and the basis for the uniformity generated by all the covers

$$
\begin{aligned}
\delta(\mathcal{Q}, a)= & \{\{(j, i)\} \mid j \in a(A) \text { and } i \in A, \exists A \in \mathbb{Q}\} \\
& \cup\{\{j\} \times A \mid j \in \beta-a(A), \exists A \in \mathbb{Q}\},
\end{aligned}
$$

where $\mathcal{Q} \in p^{\alpha} D(\alpha)$ and $a$ : $\mathbb{Q} \rightarrow \exp \beta$ is a mapping such that card $a(A)<c f \beta$ for every $A \in \mathbb{Q}$. Notice that $S_{\alpha}$ is zero-dimensional (since $p^{\alpha} D(\alpha)$ is zero-dimensional). A mapping $\pi_{\alpha}: S_{\alpha} \rightarrow p^{\alpha} D(\alpha)$ defined by $\pi_{\alpha}(j, i)=i$ is uniformly continuous since for every $\mathbb{Q} \in p^{\alpha} D(\alpha)$ we have $\pi_{\alpha}^{-1}(\mathbb{Q})=\delta(\mathbb{Q}, a)$, where $a(A)=\varnothing$ for every $A \in \mathbb{Q}$. To see that $\pi_{\alpha}: S_{\alpha} \rightarrow D(\alpha)$ is not uniformly continuous, realize that $\pi_{\alpha}^{-1}\{\{d\}, d \in D\}$ cannot be refined by any $\delta(Q, a)$ from the above described basis of $S_{\alpha}$. For such $\delta(Q, a)$ we have card $Q<\alpha$ and hence there exists $A \in \mathbb{Q}$ with card $A \geqslant 2$. Choose $j \in \beta-a(A)$. Then $\{j\} \times A \in \mathcal{S}(\mathbb{Q}, a)$, but it is not contained in any member of $\pi_{\alpha}^{-1}\{\{i\}, i \in D(\alpha)\}$. So $S$ is not $p^{\alpha}$-fine.

We will now show that $p^{\alpha} S=D(\beta) \times p^{\alpha} D(\alpha)$. Given a cover $\mathcal{P} \in S_{\alpha}$ with card $\mathscr{P}<\alpha$, let $\delta(\mathcal{Q}, a)$ be a basic cover such that $\delta(\mathcal{Q}, a)<\mathcal{P}$. For each $A \in \mathbb{Q}$, define

$$
\mathscr{P}_{A}=\bigwedge_{j \in a(A)} \pi_{\alpha}(\mathscr{P} /(\{j\} \times A)) .
$$

$\alpha=\beta^{+}$implies that card $(\mathscr{P} /(\{j\} \times A)) \leqslant \beta$ for every $j \in a(A)$. Since card $a(A)<$ $c f \beta$, we have card $\mathscr{P}_{A}<\alpha$. Define $\overline{\mathcal{P}}=\bigcup\left\{\mathscr{P}_{A} \mid A \in \mathbb{Q}\right\}$. Then $\overline{\mathcal{P}} \in p^{\alpha} D(\alpha)$ and if we 
put $D(\beta) \times \overline{\mathcal{P}}=\{\{j\} \times \bar{P} \mid j \in \beta$ and $\bar{P} \in \overline{\mathcal{P}}\}$ we obtain $D(\beta) \times \overline{\mathcal{P}} \in D(\beta) \times$ $p^{\alpha} D(\alpha)$ and $D(\beta) \times \overline{\mathcal{P}}<\mathcal{P}$.

Let $X$ be a uniform space with point-character less than or equal to $c f \beta$ such that $p^{\alpha} X=D(\beta) \times p^{\alpha} D(\alpha)$. We will prove by contradiction that $X \geqslant S_{\alpha}$. Suppose that there exists some cover $\mathfrak{V} \in X-S_{\alpha}$. Choose $\mathscr{Q} \in X$ with $\mathscr{Q}<* \mathfrak{V}$ of the form $\mathcal{Q}=\cup\left\{\{j\} \times \mathcal{Q}^{j} \mid j \in \beta\right\}$, where each $\mathcal{U}^{j}$ is a covering of $\alpha$ with the property that each point of $\alpha$ is a member of less than $c f \beta$ members of $Q^{j}$. This is possible since the point-character of $X$ is less than or equal to $c f \beta$. Then one of the following two cases is valid.

(1) There exists $I \subseteq \alpha$ such that card $I<\alpha$ and

$$
\operatorname{card}\{j \in \beta \mid(j, i) \notin \operatorname{St}(\{j\} \times I, \mathcal{Q})\}<c f \beta
$$

for every $i \in \alpha$.

(2). For every $I \subseteq \alpha$ with card $I<\alpha$ there exists $i \in \alpha$ such that

$$
\operatorname{card}\{j \in \beta \mid(j, i) \notin \operatorname{St}(\{j\} \times I, \mathcal{Q})\} \geqslant c f \beta .
$$

We will show that each case leads to a contradiction.

Case (1). For each $i \in \alpha$, define $J_{i}=\{j \in \beta \mid(j, i) \notin \operatorname{St}(\{j\} \times I, \mathcal{Q})\}$ and for each $J \subseteq \beta$ with card $J<c f \beta$ define $A_{J}=\left\{i \in \alpha \mid J_{i}=J\right\}$. Since we assume GCH, we have $\operatorname{card}\{J \subseteq \beta \mid$ card $J<c f \beta\}=\beta$, and hence $\mathbb{Q}=\left\{A_{J} \mid J \subseteq \beta\right.$ and card $J<$ $c f \beta\} \in p^{\alpha} D(\alpha)$. Define the mapping $a: \mathbb{Q} \rightarrow \exp \beta$ by setting $a\left(A_{J}\right)=J$. Since

$$
\{\operatorname{St}(i, \mathcal{Q}) \mid i \in I\} \cup\left\{\beta \in \alpha-\bigcup_{i \in I} \operatorname{St}(i, \mathscr{Q})\right\}
$$

is a uniform cover of $X$ of cardinality less than $\alpha$ and $p^{\alpha} X=D(\beta) \times p^{\alpha} D(\alpha)$, we can choose $\mathcal{S} \in D(\beta) \times p^{\alpha} D(\alpha)$ such that

$$
\delta<\{\operatorname{St}(i, \text { Q }) \mid i \in I\} \cup\left\{\beta \times \alpha-\bigcup_{i \in I} \operatorname{St}(i, \text { Q })\right\} .
$$

Then $\mathcal{S} \wedge \mathcal{S}(\mathbb{Q}, a)<\mathfrak{V}$, which contradicts the fact that $\mathfrak{V} \notin S_{\alpha}$.

Case (2). We will use transfinite induction to construct two sequences $\left\{i_{\gamma} \mid \gamma \in \alpha\right\}$ $\subseteq \alpha$ and $\left\{\varphi_{\gamma}: \mathscr{Q}_{\gamma} \rightarrow \beta \mid \gamma \in \alpha\right\}$, where $\mathscr{Q}_{\gamma}=\left\{U \in \mathcal{Q} \mid(\exists j \in \beta)\left(\exists \gamma^{\prime} \leqslant \gamma\right)\left(\left(j, i_{\gamma^{\prime}}\right) \in\right.\right.$ $U)\}$ such that for every $\delta, \gamma \in \alpha$ with $\delta \in \gamma$ we have $\varphi_{\gamma} / \mathcal{Q}_{\delta}=\varphi_{\delta}$ and there exists $j_{\delta \gamma} \in \beta$ such that

$$
\left\{\varphi_{\gamma}(U) \mid\left(j_{\delta \gamma}, i_{\gamma}\right) \in U, U \in \mathcal{Q}\right\} \cap\left\{\rho_{\gamma}(U)\left(j_{\delta \gamma}, i_{\delta}\right) \in U, U \in \mathcal{U}\right\}=\varnothing .
$$

Arbitrarily choose $i_{0} \in \alpha$ and $\varphi_{0}: \mathscr{Q}_{0} \rightarrow \beta$. Let $\gamma \in \alpha$ be an ordinal such that for every $\delta \in \gamma$ we have $i_{\gamma}$ and $\varphi_{\gamma}$ fulfilling the conditions stated above. Then (2) guarantees that there exists $i_{\gamma} \in \alpha$ such that

$$
\operatorname{card}\left\{j \in \beta \mid\left(j, i_{\gamma}\right) \notin \operatorname{St}\left(\left\{\left(j, i_{\delta}\right) \mid \delta \in \gamma\right\}, \mathcal{Q}\right)\right\} \geqslant c f \beta .
$$

Define $J_{\gamma}=\left\{j \in \beta \mid\left(j, i_{\gamma}\right) \notin \operatorname{St}\left(\left\{\left(j, i_{\delta}\right) \mid \delta \in \gamma\right\}\right.\right.$, थ $\left.)\right\}$. For every $\delta \in \gamma$, define $\varphi_{\gamma} / \mathcal{U}_{\delta}=\varphi_{\delta}$. For each $U \in \mathcal{Q}_{\gamma}-\cup_{\delta \in \gamma} \mathcal{Q}_{\delta}$ such that $\left(j, i_{\gamma}\right) \in U$ for some $j \in \beta$ $-J_{\gamma}$, choose $\varphi_{\gamma}(U)$ arbitrarily. For those $U \in \mathcal{U}_{\gamma}-\cup_{\delta \in \gamma}$ थ $_{\delta}$ for which $\left(j, i_{\gamma}\right) \in U$ for some $j \in J_{\gamma}$, we will first define $\varphi_{\gamma}(U)$ for a regular cardinal $\beta$ and then for $\beta$ singular. 
If $\beta$ is regular, then card $J_{\gamma}=c f \beta=\beta$. Since $\gamma<\alpha$ and $\alpha=\beta^{+}$, there exists a one-to-one mapping $f_{\gamma}: \gamma \rightarrow J_{\gamma}$. For every $j \in \beta-f_{\gamma}[\gamma]$ choose an arbitrary mapping $\varphi_{\gamma}^{j}:\left\{U \in \mathcal{Q} \mid\left(j, i_{\gamma}\right) \in U\right\} \rightarrow \beta-\left\{\varphi_{f_{\gamma}^{-1}(j)}(U) \mid\left(j, i_{f_{\gamma}^{-1}(j)}\right) \in U, U \in U\right\}$. This is possible, since we had taken such $Q$ that each point of $\beta \times \alpha$ is a member of less than $c f \beta=\beta$ members of $\mathcal{Q}$. Then for every $U \in \mathcal{U}$ with $\left(j, i_{\gamma}\right) \in U$ for some $j \in J_{\gamma}$, define $\varphi_{\gamma}(U)=\varphi_{\gamma}^{j}(U)$.

If $\beta$ is singular, then for every $j \in J_{\gamma}$ there exists a cardinal $\nu_{j}<\beta$ such that $\Sigma_{j \in J} \nu_{j}=\beta$. Since $\gamma<\alpha$ and $\alpha=\beta^{+}$there exists a one-to-one mapping $g_{\gamma}$ : $\gamma \rightarrow \Sigma_{j \in J} \nu_{j}$. For each $j \in J_{\gamma}$ with $g_{\gamma}^{-1}\left(\nu_{j}\right)=\varnothing$, choose an arbitrary mapping $\varphi_{\gamma}^{j}$ : $\left\{U \in \mathscr{Q} \mid\left(j, i_{\gamma}\right) \in U\right\} \rightarrow \beta$. For each $j \in J_{\gamma}$ with $g_{\gamma}^{-1}\left(\nu_{j}\right) \neq \varnothing$, choose an arbitrary mapping

$$
\begin{aligned}
\varphi_{\gamma}^{j}:\{U \in \mathscr{U} \mid & \left.\left(j, i_{\gamma}\right) \in U\right\} \\
& \rightarrow \beta-\left\{\varphi_{\delta}(U) \mid\left(j, i_{\delta}\right) \in U, g_{\gamma}(\delta) \in \nu_{j}, U \in \mathcal{Q}, \delta \in \gamma\right\} .
\end{aligned}
$$

It exists since $\operatorname{card}\left\{U \in \mathcal{U} \mid(\exists \delta \in \gamma)\left(\left(j, i_{\delta}\right) \in U\right.\right.$ and $\left.\left.g_{\gamma}(\delta) \in \nu_{j}\right)\right\} \leqslant \nu_{j} \cdot c f \beta<\beta$. Then for every $U \in \mathcal{Q}$ with $\left(j, i_{\gamma}\right) \in U$ for some $j \in J_{\gamma}$, define $\varphi_{\gamma}(U)=\varphi_{\gamma}^{j}(U)$.

Now, realize that the defined sequences $\left\{i_{\gamma} \mid \gamma \in \alpha\right\}$ and $\left\{\varphi_{\gamma} \mid \gamma \in \alpha\right\}$ have the required property: if $\beta$ is regular, define $j_{\delta \gamma}=f_{\gamma}(\delta)$ for every pair $\delta, \gamma \in \alpha$ with $\delta \in \gamma$, and if $\beta$ is singular and $g_{\gamma}(\delta) \in \nu_{j}$, define $j_{\delta_{\gamma}}=j$. Then

$$
\left\{\varphi_{\gamma}(U) \mid\left(j_{\delta \gamma}, i_{\gamma}\right) \in U, U \in \mathcal{Q}\right\} \cap\left\{\varphi_{\delta}(U) \mid\left(j_{\delta \gamma}, i_{\delta}\right) \in U, U \in \mathcal{U}\right\}=\varnothing .
$$

This completes the construction of the desired sequences.

Denote by $X^{\prime}$ the subspace of $X$ with the underlying set $\beta \times\left\{i_{\gamma}, \gamma \in \alpha\right\}$. For every $\xi \in \beta$, define

$$
W_{\xi}=\cup\left\{U \cap \mid X^{\prime} \|(\exists \gamma \in \alpha)\left(U \in \mathscr{Q}_{\gamma} \text { and } \varphi_{\gamma}(U)=\xi\right)\right\} .
$$

Since $\beta<\alpha$ and $\cup_{\xi \in \beta} W_{\xi} \supseteq \beta \times\left\{i_{\gamma}, \gamma \in \alpha\right\}$, we have $\mathscr{W}=\left\{W_{\xi} \mid \xi \in \beta\right\} \in p^{\alpha} X^{\prime}$. $p^{\alpha} X^{\prime}$ is a subspace of $p^{\alpha} X$, so there exists a partition $\mathscr{R}$ of the set $\left\{i_{\gamma} \mid \gamma \in \alpha\right\}$ having cardinality less than $\alpha$ such that $\{\{j\} \times R \mid j \in \beta, R \in R\}<\mathcal{W}$. Choose a pair $\delta, \gamma \in \alpha$ such that $\delta \in \gamma$ and $i_{\delta} \in \operatorname{St}\left(i_{\gamma}, \Re\right)$. Then there exists $\xi \in \beta$ with $\left(j_{\delta \gamma}, i_{\gamma}\right) \in$ $W_{\xi}$ and $\left(j_{\delta \gamma}, i_{\delta}\right) \in W_{\xi}$, i.e. there exists $U_{\gamma} \in \mathcal{Q}$ and $U_{\delta} \in \mathcal{Q}$ such that $\left(j_{\delta \gamma}, i_{\gamma}\right) \in U_{\gamma}$, $\left(j_{\delta \gamma}, i_{\delta}\right) \in U_{\delta}$, and $\varphi_{\gamma}\left(U_{\gamma}\right)=\varphi_{\delta}\left(U_{\delta}\right)=\xi$. But this contradicts the fact that

$$
\left\{\varphi_{\gamma}(U) \mid\left(j_{\delta \gamma}, i_{\gamma}\right) \in U, U \in \mathcal{Q}\right\} \cap\left\{\varphi_{\delta}(U) \mid\left(j_{\delta \gamma}, i_{\delta}\right) \in U, U \in \mathcal{Q}\right\}=\varnothing .
$$

REMARK. The transfinite induction construction used in the proof of Theorem II.5 is a modification of Rödl's construction in the paper $\left[\mathbf{R}_{\mathbf{1}}\right]$ mentioned in the introduction. The problem of whether Theorem II.5 remains true without the restriction on point-character is open. The author's question of whether for each infinite cardinal $\alpha$ there exists a uniform space with an underlying set of cardinality $\alpha^{+}$and point-character equal to $\alpha$ has been answered in the affirmative by Rödl in $\left[\mathbf{R}_{2}\right]$. But it is still not known whether there exists such a space with $p^{\alpha}$-modification equal to $D(\alpha) \times p^{\alpha} D\left(\alpha^{+}\right)$. 


\section{REFERENCES}

[亡̌ E. Čech, Topological spaces, Academia, Praha, 1966.

$\left[\mathbf{F}_{1}\right]$ Z. Frolik, Basic refinements of uniform spaces, General Topology and its Applications (R. A. Alo, R. W. Heath and J. Nagota, Eds.), Lecture Notes in Math., no. 378, Springer-Verlag, Berlin and New York, 1974, pp. 140-158.

$\left[\mathbf{F}_{2}\right] \ldots$, Three technical tools in uniform spaces, Seminar Uniform Spaces, 1973-74, ČSAV, Praha, 1975, pp. 3-26.

[I] J. R. Isbell, Uniform spaces, Math. Surveys, no. 12, Amer. Math. Soc., Providence, R. I., 1964.

[K] J. F. Kennison, Reflective functors in general topology and elsewhere, Trans. Amer. Math. Soc. 118 (1965), 303-315.

$\left[K_{1}\right]$ V. Kůrková, Fine and simply fine uniform spaces, Seminar Uniform Spaces 1973-74, ČSAV, Praha, 1975, pp. 127-137.

$\left[\mathbf{K}_{2}\right]$ 729-734. , Fineness in the category of uniform spaces, Proc. Colloq. on Topology, Budapest, 1978, pp.

[M] S. Mac Lane, Categories for the working mathematician, Springer-Verlag, New York, 1971.

[P] J. Pelant, Combinatorial properties of uniformities, General Topology and its Relation to Modern Analysis and Algebra IV, Prague, 1976, pp. 154-165.

[P-R] J. Pelant and V. Rödl, Coverings of infinite dimensional spaces (to appear).

$\left[\mathbf{R}_{1}\right]$ V. Rödl, Fineness in the category of all 0-dimensional uniform spaces, Seminar Uniform Spaces 1973-74, ČSAV, Praha, 1975, pp. 139-143.

$\left[\mathbf{R}_{2}\right]$, Canonical partition relation and point-character of $l_{1}$-spaces, Seminar Uniform Spaces 1976-77, pp. 79-83.

$\left[\mathbf{R}_{3}\right]$, Small spaces with a large point-character (to appear).

$\left[\mathrm{V}_{1}\right]$ J. Vilimovský, Categorial refinements and their relation to reflective subcategories, Seminar Uniform Spaces 1973-74, ČSAV, Praha, 1975, pp. 83-111.

$\left[V_{2}\right] \ldots$, Reflections on distal spaces, Seminar Uniform Spaces 1975-76, ČSAV, Praha, 1976, pp. 69-72.

Research Institute for Mathematical Machines, Prague, Czechoslovakia 